GP127 CARDIOMETABOLIC FITNESS IN 5-YEAR-OLD CHILDREN, MEASURED USING A SIMPLE STEP TEST, IS ASSOCIATED WITH BODY FAT LEVELS: FINDINGS FROM THE ROLO KIDS STUDY

Aisling A Geraghty, Eileen C O'Brien*, Mary K Horan, Jean Donnelly, Elizabeth Larkin, Fionnuala M McAuliffe. UCD Perinatal Research Centre, School of Medicine, University College Dublin, National Maternity Hospital, Dublin 2, Ireland

10.1136/archdischild-2019-epa.192

Background Fitness in early and late childhood has declined worldwide alongside the high levels of childhood obesity. Cardiovascular fitness is vital for good health and low levels have been linked with metabolic risk factors and adverse body composition in adults. However, research on fitness is limited in young children, particularly how this relates to body composition, potentially due to the lack of simple methods of estimating fitness in 5-year-old children.

Methods 272 5-year-old children from the ROLO Kids study were included in this analysis. Using a $25 \mathrm{~cm}$ step, the children stepped up and down as many times as possible for 3 minutes. A pedometer was worn to record number of steps. Participants had their heart rate measured before the test, after the 3 minutes, and monitored until heart rate returned to resting. Heart rate recovery time was calculated. Child anthropometry including height, weight, circumferences, and skinfold thickness were collected. T-tests and linear regression models were used.

Results Males had a significantly quicker heart rate recovery after completing the step test than females (113 seconds vs.129 seconds, $P<0.05)$. Participants with a slower recovery time (>105 seconds) had significantly higher sum of skinfolds compared to participants with a quicker recovery time $(P<0.03)$. Heart rate recovery remained positively associated with skinfold measurements after controlling for confounders, including child sex, age, step number, and effort rating.

Conclusion Child adiposity was positively associated with heart rate recovery, a proxy for cardiovascular fitness, using the ROLO Kids Step Test. This novel test could be used as a measure of fitness in 5-year-old children, suitable in both research and clinical settings. Replication and validation of these findings is required to provide a valid method of tracking children's fitness and health.

\section{GP128 PUBLICATION RATE OF ABSTRACTS PRESENTED AT THE IRISH PAEDIATRIC ASSOCIATION CONFERENCE OVER A 5-YEAR PERIOD}

${ }^{1}$ Kevin Gaughan*, 1,2Michael O'Grady. 'Department of Paediatrics, Midland Regional Hospital Mullingar, Mullingar, Ireland; 'Women's and Children's Health, School of Medicine, University College Dulbin, Dublin, Ireland

10.1136/archdischild-2019-epa.193

Introduction The success of a medical conference is dependent, in part, on the quality of research presented therein. Publication rates of scientific papers presented at a conference are a potentially useful surrogate for quality. Based on published data, the mean publication rate for paediatric conferences is $39 \%$. To date, no analysis of this nature has been conducted on the Irish Paediatric Association's (IPA) annual conference.

Aim This study aims to identify the rates at which abstracts presented at the IPA meeting subsequently go on for publication and what factors were associated with higher rates of publication.

Methods As $\sim 95 \%$ of publications occur within 5 years of conference presentation, abstracts from the 2008-2012 IPA conferences were selected for analysis. A PubMed/Medline search was conducted using the author's names and if required, abstract keywords. For comparability with previous studies, articles were deemed published if they were full journal articles, contained at least one similar author and reported similar outcomes. Statistical analysis, including identification of factors associated with publication success was performed using Microsoft Excel 2010.

Results Over the five-year period 584 IPA abstracts were presented and were included in our study. The mean rate of publication was $19.7 \%$ - well below the rate for other paediatric conferences. Articles were published in forty-five different journals with one-quarter were published in the Irish Medical Journal. On the basis of univariable analysis the rate of abstract publication was significantly higher for oral/podium presentations $(23 \%$ vs. $15 \%, \mathrm{p}<0.05)$, university-associated abstracts $(31 \%$ vs. $16 \%, \mathrm{p}<0.001)$ and interventional studies $(52 \%$ vs. $18 \%, \mathrm{p}<0.001)$. There was also a significantly lower rate of publication for abstracts on case reports, case series and audits compared to all other study designs (16\% vs. $24 \%$, $\mathrm{p}<0.05)$. Non-significant increases in publication rate were also observed for sample size greater than the average $(31 \%$ vs. $20 \% \mathrm{p}=0.061)$ and positive study outcome $(26 \%$ vs. $19 \%$, $\mathrm{p}=0.157)$. On multivariable analysis only university-association and interventional studies remained significantly associated with publication.

Conclusion The rate of publication of IPA abstracts was significantly below the mean for similar conferences globally. This may reflect less stringent abstract acceptance criteria but could also indicate a lack of time or support for trainees to progress their work to publication. Further exploration of these factors is planned once preliminary data collection is complete so acceptance criteria can be reviewed and trainees better supported to publish their work.

\section{GP129 THE CARIES EXPERIENCE OF CHILDREN ATTENDING THE NATIONAL PAEDIATRIC CRANIOFACIAL CENTRE (NPCC), DUBLIN, IRELAND}

${ }^{1}$ Mairéad Kelly*, ${ }^{2}$ Eleanor McGovern, ${ }^{2}$ Dylan Murray. 'Dublin Dental University Hospital, Dublin, Ireland; ${ }^{2}$ Temple Street Children's University Hospital, Dublin, Ireland

\subsection{6/archdischild-2019-epa.194}

Introduction Children with craniofacial conditions would be considered to be at a higher risk of and from dental caries. Despite this high risk, there is limited data in the literature reporting on the dental caries experience of these children.

For the last number of years children attending the National Paediatric Craniofacial Centre (NPCC) have been enrolled in the tertiary paediatric dental services by their first birthday. They are also referred to primary services for ongoing prevention of dental disease.

The objective of this review was to determine the caries experience of a cohort of children attending the NPCC over an 18-month timeframe.

Methods The World Health Organisation examination criteria were used with the inclusion of visual caries. Dental caries was recorded using the $\mathrm{dmft}$ (decayed, missing and filled 
primary teeth) index (WHO, 1987). For the purposes of our study, a single, calibrated examiner (EMcG) carried out clinical examination and recorded the $\mathrm{dmft}$ score in the dental record of each child.

Results The dmft of the 33 children aged between 13 months and 8 years of age attending the NPCC was obtained, compiled in a dataset, and analysed. For all children in the cohort $(\mathrm{n}=33)$, the average dmft was 1.2 . Of the 33 children evaluated, $75.8 \%$ were caries free $(\mathrm{dmft}=0)$ and $97 \%$ were dentally fit $(\mathrm{dt}=0)$. The overall treatment index for this cohort was $94.7 \%$.

To compare to national values, the same parameters for 5year olds were evaluated $(\mathrm{n}=12)$. The mean $\mathrm{dmft}$ for this group was 1.3. Of this group, $66.67 \%$ were caries free, $91.67 \%$ were dentally fit and the overall treatment index was $87.5 \%$. According to the National Survey, the dmft of 5-yearolds in the general population was 1.3 .

Conclusion Children with craniofacial conditions may be at higher risk of dental caries than the general child population for a variety of reasons. The present study details the caries experience of children attending a single centre, the NPCC, across a broad age range. Our findings suggest that although the overall $\mathrm{dmft}$ of 5 -year-old children in the study was equivalent to children of the same age in the National Survey, the treatment index of the children attending the NPCC far exceeds that of the general population (Whelton et al., 2002). It is reassuring to observe that across all age groups studied, a high proportion of the children in our cohort were dentally fit.

\section{GP130 ABSTRACT WITHDRAWN}

\section{GP131 HEALTH CARE TRANSITION FOR ADOLESCENTS AND YOUNG ADULTS WITH LONG-TERM CONDITIONS: QUALITATIVE STUDY OF PATIENTS, PARENTS AND HEALTHCARE PROFESSIONALS' EXPERIENCES IN IRELAND}

${ }^{1}$ Imelda Coyne*, ${ }^{1}$ Aisling Sheehan, ${ }^{1}$ Emily Heery, Alison While ${ }^{2} .{ }^{1}$ Trinity College Dublin, Dublin, Ireland; ' ${ }^{2}$ ing's College London, London, UK

\subsection{6/archdischild-2019-epa.195}

Transition of adolescents and young adults within healthcare services has become increasingly important as more children are surviving into adulthood with long-term conditions. Yet limited empirical evidence exists regarding transition experiences. Transition is not simply about a physical transfer to another service as involves multiple components and medical, psychosocial, developmental, educational and vocational needs. Successful transition to adult services requires a person-centred, developmental, psychosocial, flexible, multidisciplinary, coordinated approach.

We undertook the first Irish study to examine needs and perspectives in relation to healthcare transition for adolescents and young adults with the following long-term conditions: diabetes, cystic fibrosis, and congenital heart disease. Using a qualitative inductive approach we carried out semistructured interviews with adolescents and young adults aged 14-25 years $(n=47)$, parents $(n=36)$ and health professionals $(n=34)$ which was part of a larger mixed-methods study on transition. We accessed adolescents before transition and then a different sample who had made the transition within same disease group. The sample was obtained from two children's hospitals and four general hospitals in Ireland. Ethical approval was obtained from relevant ethics committees.

Findings revealed that transition occurred between the ages of 16 and early 20s depending on the service. None of the hospitals had a transition policy and transition practices varied considerably. Adolescents worried about facing the unknown, communicating and trusting new staff and self-management. Transition process was smooth for some young adults, whilst others experienced very abrupt transfer. Parents desired greater involvement in the transition process with some perceiving a lack of recognition of the importance of their role. In paediatric services, nurses made great efforts to engage adolescents who struggled with treatment adherence and clinic attendance. Whereas after transfer, little effort was made to engage young adults if there were lapses in care, as this was generally considered the young person's prerogative.

This study has revealed that transitioning to adult care is challenging for many AYAs, irrespective of their condition, due to unmet needs in relation to information, transition preparation, and development of self-management skills. The degree to which the shift in responsibility had occurred prior to transition to adult services appeared to influence successful transition to new roles for adolescents and young adults and their parents. Interventions need to address the renegotiation of responsibility between AYAs and parents using a developmental framework over a period of time rather than a one-off 'chat'.

\section{GP132 DEVELOPING RESEARCH CAPACITY: BASELINE SURVEY IDENTIFYING RESEARCH ACTIVITY, SKILLS AND SUPPORTS FOR NURSES IN CHILDREN'S HOSPITALS IN IRELAND}

${ }^{1}$ Norma O'Keeffe, ${ }^{1}$ Anne Marie Cullinane, 'Louise Greensmith, ' ${ }^{1}$ Shirley Bracken, ${ }^{1}$ Norma O'Shaughnessy, ${ }^{2}$ Caitriona Dennehy, ${ }^{3}$ Hazel Smith, ${ }^{4}$ Veronica Lambert ${ }^{*} .{ }^{1}$ Children's Health Ireland (Temple Street Children's University Hospital), Dublin, Ireland; ${ }^{2}$ Children's Health Ireland (Tallaght University Hospital), Dublin, Ireland; ${ }^{3}$ Children's Health Ireland (Our Lady's Children's Hospital Crumlin), Dublin, Ireland; ${ }^{4}$ School of Nursing and Human Sciences, Dublin City University, Dublin, Ireland

\subsection{6/archdischild-2019-epa.196}

Background Children's healthcare in Ireland is currently undergoing radical transformation with the build of a new National Children's Hospital to integrate services delivered by three existing children's hospitals. The new Model of Care for Irish Paediatrics endorses research-led enquiry and translation as critical to providing excellence in clinical practice. This brings its challenges with a recognised critical deficit in the number of individuals with the skills, ambition and time to lead major research projects. The aim of this study was to identify baseline data on research activity, skills and supports for nurses in children's hospitals.

Methods A cross-sectional survey was conducted in 2018 across three children's hospitals in Ireland using a clinical nursing research questionnaire developed through adaptation of two previous established questionnaires investigating research activities, skills/abilities and supports among health care providers. All registered nurses employed in the three paediatric hospital study sites were eligible to participate. 